\title{
Elevated serum LAMC2 is associated with lymph node metastasis and predicts poor prognosis in penile squamous cell carcinoma
}

This article was published in the following Dove Press journal:

Cancer Management and Research

\section{Qiang-Hua Zhou, ${ }^{1,2, *}$ Chuang-Zhong Deng, ${ }^{1, *}$ Jie-Ping Chen,' Kang-Bo Huang, ${ }^{1,2}$ Ting-Yu Liu, ${ }^{1,2}$ Kai Yao, ${ }^{1,2}$ Zhuo-Wei Liu, ${ }^{1,2}$ Zi-Ke Qin, ${ }^{1,2}$ Yong-Hong Li, ${ }^{1,2}$ Sheng-Jie Guo, ${ }^{1,2}$ Yun- Lin Ye, ${ }^{1,2}$ Fang-Jian Zhou, ${ }^{1,2}$ Wenlin Huang, 1,3 Ran-Yi Liu,' Hui Han ${ }^{1,2}$ \\ 'State Key Laboratory of Oncology in South China, Collaborative Innovation Center of Cancer Medicine, Sun Yat-sen University Cancer Center, Guangzhou, China; ${ }^{2}$ Department of Urology, Sun Yat-sen University Cancer Center, Guangzhou, China; ${ }^{3}$ Guangdong Provincial Key Laboratory of Tumor Targeted Drugs, Guangzhou Enterprise Key Laboratory of Gene Medicine, Guangzhou Doublle Bioproducts Co. Ltd., Guangzhou, China}

*These authors contributed equally to this work

Correspondence: Hui Han State Key Laboratory of Oncology in South China and Collaborative Innovation Center of Cancer Medicine, Sun Yat-sen University Cancer Center, Guangzhou, China

Tel +862087343862

Email hanhui@sysucc.org.cn

Ran-Yi Liu

Sun Yat-sen University Cancer Center, 65 I Dong-feng Road East, Guangzhou 510060, China

Tel +862087342303

Email liury@sysucc.org.cn
Purpose: Molecular biomarkers, especially serologic factors, have been widely applied in cancer diagnosis and patient follow-up. However, there are few valuable prognostic factors in penile squamous cell carcinoma (PSCC). Here, the authors investigated whether laminin gamma 2 (LAMC2) expression, especially serum LAMC2 (sLAMC2) level, was a suitable prognostic factor that could aid in the prediction of survival in PSCC.

Patients and methods: This study included 114 PSCC patients. Reverse transcriptionquantitative polymerase chain reaction, Western blotting, and immunohistochemistry were performed to detect LAMC2 expression; enzyme-linked immunosorbent assays were used to test sLAMC2 concentration; and a Transwell assay and an in vivo experiment in nude mice were used to test PSCC cell migration, invasion, and metastasis. The chi-squared test was used to analyze the association between LAMC2 level and clinical parameters, the Cox proportional hazards regression model was used to evaluate the hazard ratio for death, and Kaplan-Meier analysis with a log-rank test was used for the survival analysis.

Results: LAMC2 was overexpressed in PSCC tissues, and the LAMC2 expression level was higher in metastatic lymph node (LN) tissues than in primary cancer tissues; moreover, the LAMC2 levels in primary cancer tissues and SLAMC2 were higher in patients with LN metastasis than in those without LN metastasis. Upregulated LAMC2 facilitated the migration, invasion, and epithelial-to-mesenchymal transition of PSCC cells in vitro and promoted LN metastasis of PSCC cells in nude mice. Elevated LAMC2 levels were strongly correlated with advanced clinicopathologic parameters, especially LN metastasis, in PSCC patients and predicted shorter disease-specific survival. The predictive value of sLAMC2 is superior to that of C-reactive protein and squamous cell carcinoma antigen previously reported in PSCC patients, and a stratification analysis revealed that the level of SLAMC2 had a higher predictive value for disease-specific survival in early penile cancer (especially at the $\mathrm{N}_{0 / \mathrm{X}}$ stage) than in later-stage penile cancer.

Conclusion: These findings suggest that SLAMC2 is a potential serologic prognostic marker in PSCC and could aid in risk stratification in early-stage PSCC patients.

Keywords: penile neoplasms, serum markers, laminin, gamma 2, neoplasm metastasis

\section{Introduction}

Penile squamous cell carcinoma (PSCC) is a relatively rare malignant tumor worldwide; however, in developing countries, it has a much higher incidence and accounts for up to $2 \%$ of malignancies in men. ${ }^{1}$ The major risk factors mainly comprise human papilloma virus infection, phimosis, and poor hygiene. ${ }^{2}$ The involvement of local lymph nodes (LNs) is the major prognostic factor in PSCC. ${ }^{3-5}$ The main treatment for PSCC is currently a partial or radical penectomy with or without bilateral inguinal 
lymphadenectomy, along with radiation, chemotherapy, and targeted therapy. ${ }^{1,6-9}$

Some classical serum biomarkers (such as CEA, AFP, PSA, and CA-199) are clinically useful in many cancers ${ }^{10-12}$; however, they are not suitable in penile cancer. Serum $\mathrm{C}$-reactive protein (CRP) and squamous cell carcinoma antigen (SCC-Ag) were reported to be prognostic markers in PSCC. ${ }^{13-15}$ However, large-scale investigations are required to assess the clinical application of these markers. The discovery of more serologic biomarkers would be helpful in guiding treatment and predicting prognosis in PSCC.

Together with laminin alpha 3 and laminin beta 3, laminin gamma 2 (LAMC2) composes the heterotrimeric basement membrane component laminin 332 and plays important physiologic and pathologic roles through its involvement in cellular migration and adhesion, especially in the processes of tumor invasion and metastasis. ${ }^{16}$ It has been reported that the overexpression of $L A M C 2$ is associated with a poor prognosis in numerous cancers, ${ }^{17-19}$ and cytoplasmic accumulation of LAMC2, as opposed to its extracellular deposition in the basement membrane, is correlated with cancer metastasis and poor prognosis. ${ }^{18}$ Interestingly, elevated LAMC2 levels have also been detected in peripheral blood, indicating that LAMC2 could be a novel cancer biomarker. ${ }^{12,19}$ However, the expression level of LAMC2 and its clinical significance are unknown in PSCC.

In this study, we found that LAMC2 was overexpressed in PSCC tissues and facilitated the metastasis of cancer cells and that elevated serum LAMC2 (sLAMC2) levels predicted poor disease-specific survival (DSS) and could aid in risk stratification of early-stage PSCC patients.

\section{Patients and methods}

\section{Patients and samples}

One hundred fourteen patients newly diagnosed with PSCC were included in this study. All patients underwent a partial or radical penectomy with or without bilateral inguinal lymphadenectomy at Sun Yat-sen University Cancer Center (SYSUCC) between 2009 and 2015. Surgical specimens were collected and then paraffin-embedded or cryopreserved with liquid nitrogen. The study protocol was approved by the Institutional Ethics Board of SYSUCC, and written informed consent was obtained from each person. The pathologic TNM stage was redetermined by two pathologists according to the 2018 American Joint Committee on Cancer TNM staging system for PSCC. The clinicopathologic characteristics are presented in Table S1.

\section{sLAMC2, SCC-Ag, and CRP measurements}

sLAMC2 levels were measured using an enzyme-linked immunosorbent assay kit (USCN Life Sciences, Wuhan, China) according to the manufacturer's protocol, and the serum SCC-Ag and CRP concentrations were measured at the Department of Molecular Diagnosis, SYSUCC, before surgery. High or low levels of sLAMC2, CRP, and SCC-Ag were distinguished by their corresponding cutoff values (sLAMC2: $108 \mathrm{ng} / \mathrm{mL}$; CRP: $5.0 \mathrm{mg} / \mathrm{mL}$; and SCC-Ag: $1.5 \mathrm{ng} / \mathrm{mL}$ ). The cutoff values of sLAMC2 and CRP were obtained by receiver operating characteristic (ROC) curve analysis, whereas the cutoff value for SCC-Ag followed the clinical guidelines.

\section{Immunohistochemistry (IHC) assays}

IHC assays were conducted as previously described to detect LAMC2 and E-cadherin expression in tissues. Briefly, 4- $\mu \mathrm{m}$-thick formalin-fixed paraffin-embedded tissue sections were deparaffinized and rehydrated, followed by antigen retrieval and endogenous peroxidase inactivation. ${ }^{20,21}$ After blocking, the slides were incubated with LAMC2 (SigmaAldrich Co., St. Louis, MO, USA) or E-cadherin antibody (Cell Signaling Technology [CST], Danvers, MA, USA), followed by horseradish peroxidase (HRP)-conjugated secondary antibody (CST), and were then visualized using a peroxidase Envision kit (Dako, Carpinteria, CA, USA). All slides were independently evaluated by two pathologists to determine the percentage of cancer cells with positive staining. Cells with cytoplasmic staining were considered to be positive for LAMC2, while cells with membrane staining were positive for E-cadherin (Figure S1). According to the definitions used in previous reports, a high level of LAMC2 expression in tumor tissues (tLAMC2) was defined as more than $30 \%$ of the tumor cells staining positive for LAMC2, ${ }^{17,22}$ while a low level of E-cadherin expression was defined as less than $90 \%$ of the tumor cells staining positive for E-cadherin. ${ }^{23}$

\section{Reverse transcription and quantitative polymerase chain reaction (RT-qPCR)}

LAMC2 mRNA levels in tumor tissues were detected by RT-qPCR. Briefly, total RNA was extracted and reverse transcribed using PrimeScript ${ }^{\mathrm{TM}}$ Master Mix (TaKaRa, Dalian, China), and quantitative PCR was performed using SYBR ${ }^{\circledR}$ Premix Ex Taq ${ }^{\mathrm{TM}}$ (TaKaRa) with specific primers (Table S2). The relative expression of genes is presented as $2^{-\Delta \mathrm{Ct}}$ (normalized against $G A P D H$ expression). ${ }^{24,25}$ 


\section{Western blotting (WB)}

WB was performed as previously described ${ }^{26,27}$ to analyze protein levels in cells and frozen tissues. Briefly, protein samples were routinely extracted, quantified, separated by sodium dodecyl sulfate-polyacrylamide gel electrophoresis and transferred to polyvinylidene difluoride membranes. After blocking, the membranes were incubated with LAMC2- (Sigma-Aldrich Co.), E-cadherin-, N-cadherin-, ZEB-1-, ZO-1-, vimentin-, or GAPDH-specific antibodies (CST) (1:1000-2000), followed by incubation with an HRP-conjugated secondary antibody (CST). The bands were visualized with Clarity Western ECL Substrate and detected through a ChemiDoc Touch Imaging System (Bio-Rad Laboratories Inc., Hercules, CA, USA).

\section{Cell lines and establishment of stable cell lines}

PSCC cell lines (Penl1, Penl2, and 149RCa) were established in our laboratory. ${ }^{28-30} \mathrm{HaCaT}$ and $293 \mathrm{~T}$ cells were obtained from the CAS Type Culture Collection of the Chinese Academy of Sciences (Shanghai, China). All cell lines were maintained in Dulbecco's modified Eagle medium supplemented with $10 \%$ fetal bovine serum (Thermo Fisher Scientific, Waltham, MA, USA).

Stable cell lines with overexpressing or silenced LAMC2 were established according to the methods described previously. ${ }^{31,32}$ Briefly, LAMC2 cDNA was cloned into the pCDHEF1-CMV-MCS-T2A-Puro vector (System Biosciences, Palo Alto, CA, USA), and LAMC2 shRNAs (Table S3) were inserted into the pLko.1 vector (Sigma-Aldrich Co., St. Louis, MO, USA). Recombinant lentiviruses were packaged in $293 \mathrm{~T}$ cells and used to construct the stable cell lines.

\section{Cell proliferation assay}

PSCC cells were cultured in 96-well plates for the indicated times, and cell proliferation was then analyzed using the Cell Counting Kit-8 assay (Dojindo, Japan) and represented by the optical density at $450 \mathrm{~nm}\left(\mathrm{OD}_{450 \mathrm{~nm}}\right) \cdot{ }^{33}$

\section{Migration and invasion assay}

Transwell assays were used to evaluate the migration and invasion abilities of PSCC cells, as previously described..$^{34,35}$ Briefly, $1 \times 10^{5}$ Penl 2 cells, $2 \times 10^{5}$ Penl 1 cells, or $2 \times 10^{5} 149$ $\mathrm{RCa}$ cells were seeded into Boyden chambers $(8 \mu \mathrm{m}$ pore) without or with Matrigel coating (BD Biosciences, San Jose, CA, USA). After 18 or 24 hours of incubation for the migration or invasion assays, respectively, cells on the lower surface of the filter were fixed, stained, and counted under a microscope. All assays were performed in duplicate and were repeated three times.

\section{Animal experiments}

Animal experiments were approved by the SYSUCC Institutional Animal Care and Use Committee following the Animal Welfare and Rights in China. Male BALB/c nude mice (4-5 weeks old; SLRC Laboratory Animals, Shanghai, China) were used to generate an inguinal LN metastasis model, as described previously. ${ }^{31}$ Two million cells were injected into the foot pads of the mice ( $\mathrm{n}=10 /$ group). The mice were killed after 10 12 weeks, and inguinal LNs were examined.

\section{Statistical analysis}

All statistical analyses were performed using the SPSS statistical software: the chi-squared test was used for the correlation analyses, the Cox proportional hazards regression model was used to calculate the HR for death, KaplanMeier analysis with a log-rank test was used for the survival analysis, and ROC curve analysis was used to calculate the optimum cutoff values. Statistical significance was defined as $P<0.05$.

\section{Results}

\section{LAMC2 is upregulated in the cancer tissues and serum of PSCC patients}

$L A M C 2$ expression was significantly upregulated at the mRNA level (29/30, Figure 1A) and protein level (14/15, Figure 1B) in the PSCC tissues compared to that in paired normal epithelial tissues. Moreover, $L A M C 2$ expression was higher in LN metastatic lesions than in primary cancer tissues (Figure 1C). There was more cytoplasmic accumulation of LAMC2 in PSCC tissues than normal tissues, and the ratio of local PSCC tissues expressing high level of LAMC2 is higher in patients with LN metastasis (LNMP) than in LN metastasis-free patients (MFP) (Figure 1D).

The concentration of sLAMC2 was markedly higher in PSCC patients with LN metastasis than those in MFP and healthy age-matched volunteers, while no differences were found between the two latter groups (Figure 1E). Moreover, the sLAMC2 level likely gradually increased with more advanced $\mathrm{N}$ stage (Figure 1E). These data suggest that overexpression of $L A M C 2$ is mainly related to $\mathrm{LN}$ metastasis in PSCC.

\section{LAMC2 promotes migration, invasion, and $L N$ metastasis of PSCC cells}

$L A M C 2$ was expressed at a significantly higher level in PSCC cells than in the immortalized human keratinocyte $\mathrm{HaCaT}$ 

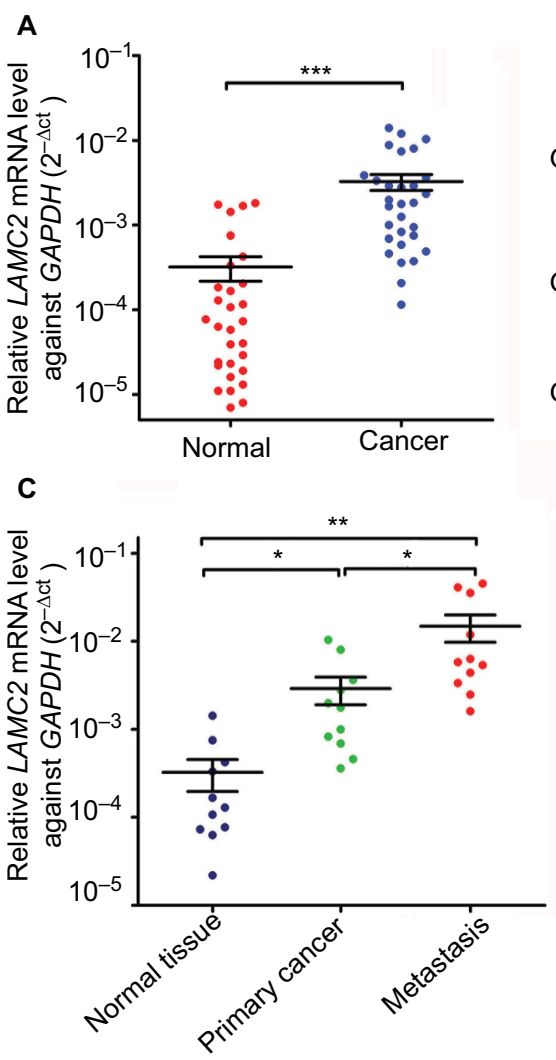

D

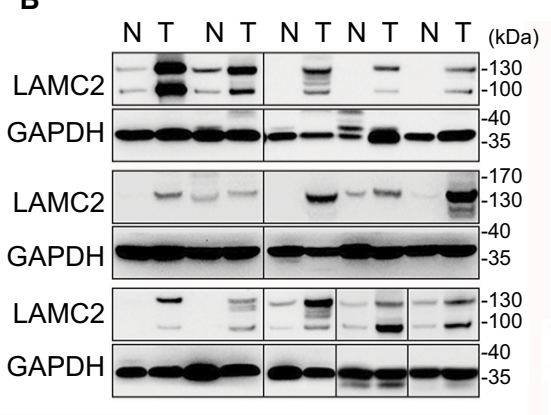

E

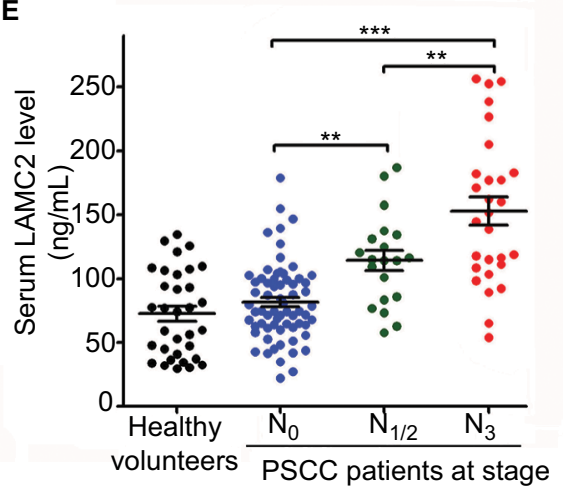

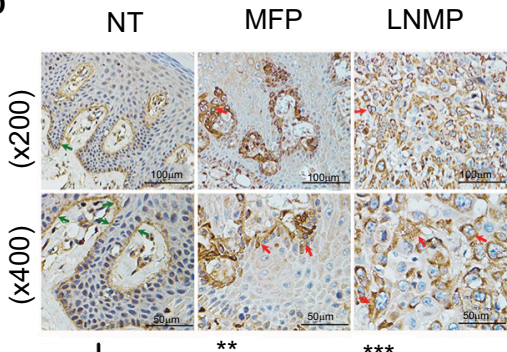

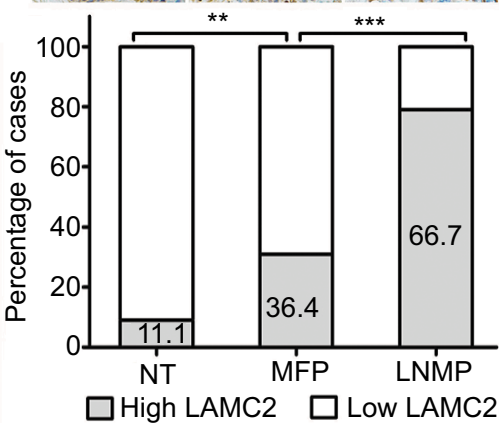

Figure I LAMC2 is overexpressed in PSCC tissues, especially in those with LN metastasis.

Notes: (A) RT-qPCR $(n=30)$ and (B) WB analysis $(n=15)$ for LAMC2 expression in paired PSCC and normal epithelial tissues (GAPDH was used as the loading control for WB analysis). (C) RT-qPCR analysis for LAMC2 expression in II paired PSCC, LN metastatic focuses, and normal epithelial tissues. (D) IHC assays for LAMC2 expression in normal epithelial tissues (NT, $n=45$ ), primary cancer tissues from LNMP $(n=48)$, and primary cancer tissues from MFP ( $n=66$ ). Upper: representative IHC images (the green arrows indicate positive staining in the basement membrane, and the red arrows indicate positive staining in cancer cells); Lower: the percentages of samples with high or low LAMC2 levels. (E) The serum LAMC2 level in healthy volunteers and PSCC patients. Student's $t$-test or analysis of variance was used to compare the differences between groups. $* P<0.05, * * P<0.01, * * * P<0.001$.

Abbreviations: LAMC2, laminin gamma 2; LN, lymph node; LNMP, patients with LN metastasis; MFP, LN metastasis-free patients; PSCC, penile squamous cell carcinoma; RT-qPCR, reverse transcription and quantitative polymerase chain reaction; WB, Western blotting; IHC, immunohistochemistry.

cells (Figure 2A), in accordance with the findings in clinical cases. Penl1 and Penl 2 cells showed much higher LAMC2 expression than 149RCa cells.

Further investigation revealed that $L A M C 2$ overexpression significantly increased the migration and invasion of 149RCa cells (Figure 2A, B), whereas LAMC2 silencing significantly inhibited the migration and invasion of Penl1 and Penl2 cells (Figure 2A, C). Moreover, LAMC2 knockdown significantly reduced the ability of Penl 2 cells to metastasize to inguinal LN (Figure 2D). However, overexpression and knockdown of $L A M C 2$ did not obviously affect the proliferation of PSCC cells (Figure S2). Taken together, these results indicate that LAMC2 may play important roles in promoting PSCC cell migration, invasion, and LN metastasis.

\section{LAMC2 promotes epithelial-to- mesenchymal transition (EMT) of PSCC cells}

The expression of the epithelial markers E-cadherin and ZO1 was found to be downregulated in LAMC2-overexpressing
149RCa cells and upregulated in LAMC2-silenced Penl1 and Penl 2 cells compared to their corresponding control cells (Figure 2E); conversely, the expression of the mesenchymal markers N-cadherin and ZEB1 decreased in $L A M C 2$-silenced Penl1 and Penl 2 cells and increased in LAMC2-overexpressing 149RCa cells (Figure 2E). The clinical data analysis revealed that a high level of tLAMC2 displayed a close correlation with low expression of E-cadherin in PSCC tissues and that a high sLAMC2 level was also correlated with a low E-cadherin level (Figure 2F). These data suggest that LAMC2 may promote EMT and then facilitate PSCC metastasis.

\section{LAMC2 expression is closely associated with the outcomes of PSCC patients}

As LAMC2 promotes LN metastasis, the relationship between $L A M C 2$ expression and clinicopathologic parameters was investigated in 114 PSCC patients, and the prognosis of these patients was estimated. The results showed that a high level of tLAMC2 was strongly correlated with pathologic grade and $\mathrm{N}$ stage but not with T stage (Table 1); 
A

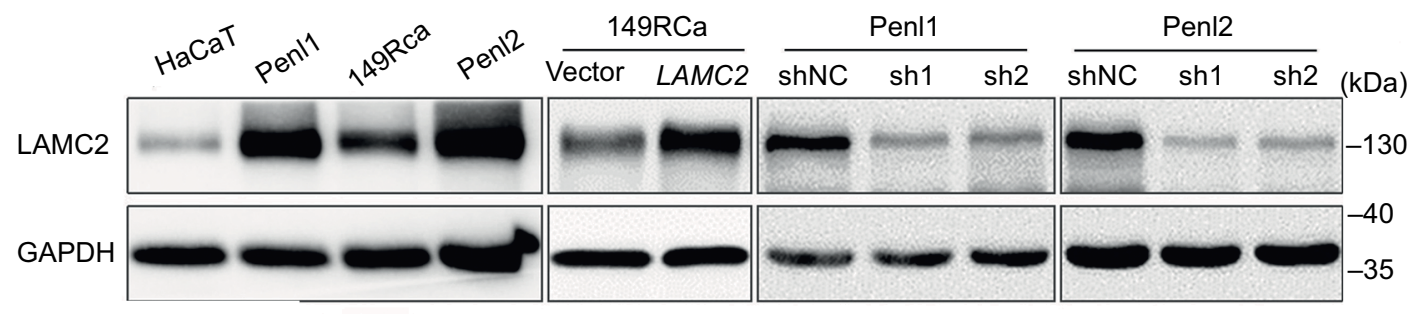

B
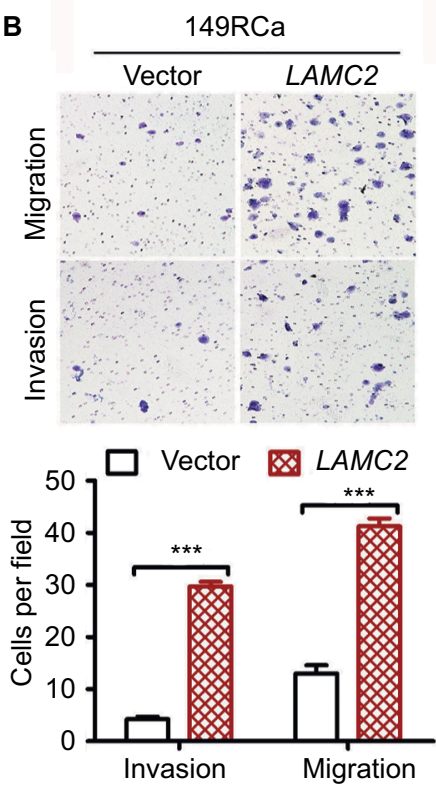

C

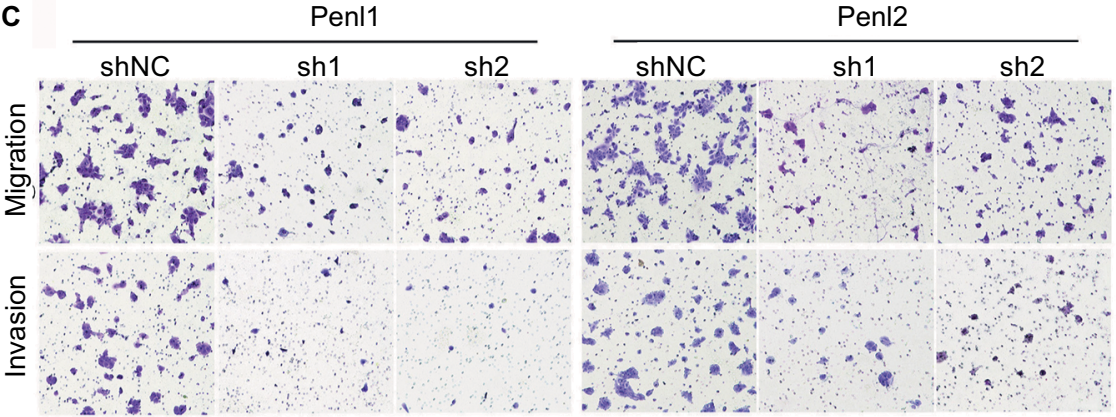

$\square$ shNC 图 sh1 囲 sh2
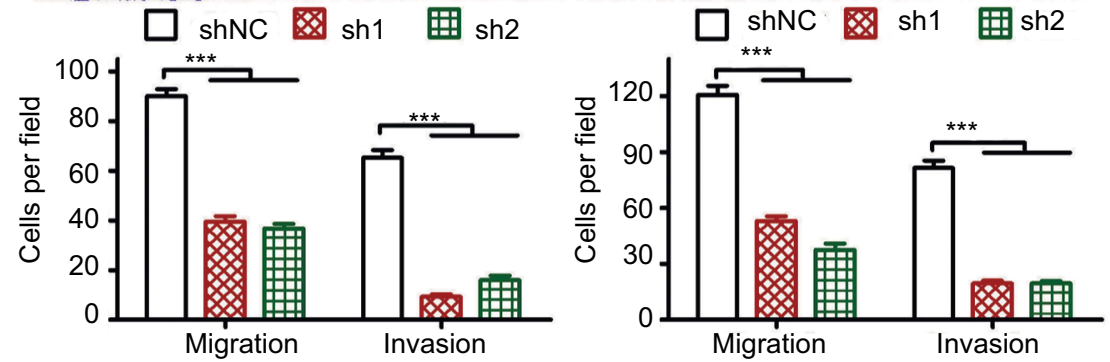

D

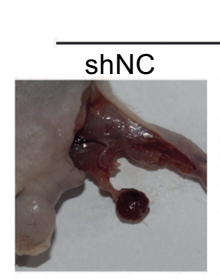

Penl2

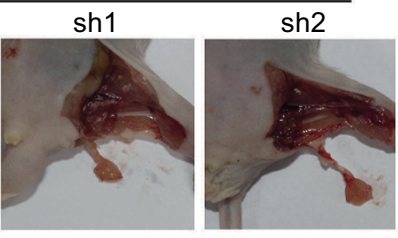

sh1

sh2 shNC

(

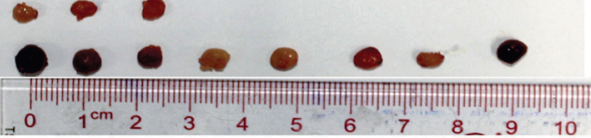

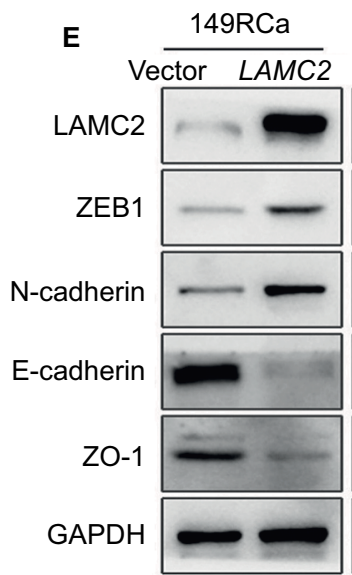

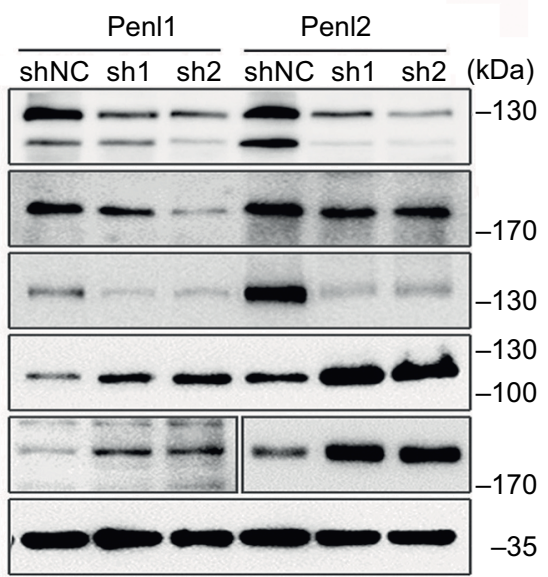

$\mathbf{F}$

\begin{tabular}{ccccccccc}
\hline & & \multicolumn{3}{c}{ tLAMC2 } & & \multicolumn{3}{c}{ sLAMC2 } \\
\cline { 3 - 5 } \cline { 7 - 9 } & & Low & High & $p$ value & & Low & High & $p$ value \\
\hline \multirow{2}{*}{ E-cadherin } & Low & 24 & 34 & $0.039^{*}$ & & 30 & 28 & $0.005^{* *}$ \\
& High & 34 & 22 & & & 43 & 13 & \\
\hline
\end{tabular}

Figure 2 LAMC2 promotes the migration, invasion, and LN metastasis of PSCC cells by inducing EMT.

Notes: After stably overexpressing or silencing LAMC2, PSCC cells were assessed for migration, invasion, and LN metastatic activity using Transwell assays or an inguinal LN metastasis model in BALB/c nude mice. (A) WB analysis for LAMC2 expression. (B, C) Transwell assays to determine the effect of LAMC2 on migration and invasion of PSCC cells (B: LAMC2-overexpressed; C: LAMC2-silenced). Upper, representative images; lower, bar charts indicating the average numbers of migrated/invaded cells in triplicate experiments. (D) The effect of LAMC2 on LN metastasis using an inguinal $L N$ metastasis model in BALB/c nude mice ( $n=10 /$ group). Upper, representative images of inguinal LN metastatic nodules; lower, the array of metastatic LNs. (E) WB analysis for the expression of EMT-related proteins. (F) Association between LAMC2 expression and E-cadherin expression in clinical PSCC tissues. Student's $t$-test or analysis of variance was used to compare the differences between groups, and the chi-squared test was used to analyze the relationship between variables. $* P<0.05$, $* * P<0.01$, $* * * P<0.001$.

Abbreviations: EMT, epithelial-to-mesenchymal transition; LAMC2, laminin gamma 2; LN, lymph node; PSCC, penile squamous cell carcinoma; sLAMC2, serum LAMC2 level; tLAMC2, tissue LAMC2 level; WB, Western blotting; sh I, sh2: LAMC2 shRNAs; shNC, negative control shRNA. 
the tLAMC2 level was also correlated with the sLAMC2 level, which was associated not only with pathologic grade and $\mathrm{N}$ stage but also with $\mathrm{T}$ stage (Table 1 ).

The univariate Cox regression analysis suggested that the LAMC2 (both tLAMC2 and sLAMC2), SCC-Ag, and CRP levels, along with $\mathrm{T}$ stage, $\mathrm{N}$ stage, and pathologic grade, showed significant prognostic value for survival (Table S4). The Kaplan-Meier analysis suggested that tLAMC2, sLAMC2, SCC-Ag, and CRP levels were significantly correlated with DSS in PSCC; however, patients with a high level of sLAMC2 had markedly lower 3-year survival rates than those with a high level of tLAMC2, SCC-Ag, or CRP (sLAMC2 42.7\% vs tLAMC2 62.0\%, SCC-Ag 55.8\%, or CRP 58.0\%) (Figure 3A-D).

Furthermore, we combined $\mathrm{T} / \mathrm{N}$ stage and pathologic grade with each potential biomarker in a multivariate Cox regression analysis and found that among the levels of tLAMC2, sLAMC2, SCC-Ag, and CRP, only sLAMC2 level was an independent prognostic indicator for DSS in PSCC patients (Table 2 and Table S5). These data indicate that SLAMC2 is a more important risk factor for DSS in PSCC patients than tLAMC2, SCC-Ag, or CRP.

\section{sLAMC2 had a higher predictive value for DSS in early-stage penile cancer}

A subgroup survival analysis was performed to evaluate the predictive value of sLAMC2 for the survival of patients strati- fied by $\mathrm{T}$ stage, pathologic grade, and $\mathrm{N}$ stage. The results showed that a high sLAMC2 level indicated a high risk of death in patients at a less-advanced $\mathrm{T}$ stage $\left(\mathrm{T}_{1 / \mathrm{a}}\right.$ or $\left.\mathrm{T}_{2}\right)$ and without $\mathrm{LN}$ metastasis $\left(\mathrm{N}_{0 / \mathrm{X}}\right)$ but did not in those with $\mathrm{T}_{3 / 4}$ stage or $\mathrm{N}_{+}$stage disease, while there were no differences in the prognostic value of sLAMC2 among the different pathologic grade classifications (Table 3). Kaplan-Meier analysis demonstrated that sLAMC2 level was significantly associated with DSS in PSCC patients at the $\mathrm{T}_{1 / \mathrm{a}}, \mathrm{T}_{2}$, and $\mathrm{N}_{0 / \mathrm{X}}$ stages, and the cumulative 3-year survival rates were markedly lower in the high-LAMC2 group than in the low-LAMC2 group $\left(\mathrm{T}_{1 / \mathrm{a}}: 50.0 \%\right.$ vs $93.5 \% ; \mathrm{T}_{2}: 47.4 \%$ vs $91.0 \%$; and $\mathrm{N}_{0 / \mathrm{X}}: 80 \%$ vs $91.1 \%$ ) (Figure $3 \mathrm{E}-\mathrm{G})$.

\section{Discussion}

Serologic markers are more accessible for detection compared to the pathologic parameters, although pathologic indicators are the most important prognostic factors, especially the $\mathrm{N}$ stage. ${ }^{3}$ Serum markers, such as AFP, CEA, PSA, and CA19.9, have been widely applied in clinical practice as adjuncts to clinicopathologic parameters for monitoring tumor progression and predicting survival. ${ }^{10-12}$ However, few valuable prognostic serologic factors have been identified in PSCC. Although serum CRP and SCC-Ag levels have been reported to predict postoperative recurrence, progression, and prognosis in PSCC, ${ }^{13-15}$ their prognostic values need to be validated by large-scale investigations.

Table I Association between LAMC2 expression level and the clinicopathologic characteristics of PSCC patients

\begin{tabular}{|c|c|c|c|c|c|c|c|c|}
\hline \multirow[t]{2}{*}{ Variables } & & \multirow{2}{*}{$\begin{array}{l}\text { No. of } \\
\text { patients }\end{array}$} & \multicolumn{3}{|c|}{ tLAMC2 } & \multicolumn{3}{|c|}{ SLAMC2 } \\
\hline & & & Low & High & $P$-value & Low & High & $P$-value \\
\hline \multirow[t]{2}{*}{ Age (years) } & $<54$ & 57 & 28 & 29 & 0.708 & 35 & 22 & 0.558 \\
\hline & $\geq 54$ & 57 & 30 & 27 & & 38 & 19 & \\
\hline \multirow[t]{2}{*}{ Phimosis } & No & 33 & 15 & 18 & 0.460 & 18 & 15 & 0.178 \\
\hline & Yes & 81 & 43 & 38 & & 55 & 26 & \\
\hline \multirow[t]{2}{*}{ T stage } & $T_{1 / a}$ & 37 & 22 & 15 & 0.204 & 31 & 6 & $0.002 * *$ \\
\hline & $\mathrm{T}_{2-4}$ & 77 & 36 & 41 & & 42 & 35 & \\
\hline \multirow[t]{2}{*}{ Pathologic grade } & $\mathrm{G}_{1}$ & 70 & 43 & 27 & $0.004^{* *}$ & 51 & 19 & $0.013^{*}$ \\
\hline & $\mathrm{G}_{2-3}$ & 44 & 15 & 29 & & 22 & 22 & \\
\hline \multirow[t]{2}{*}{$N$ stage } & $\mathrm{N}_{0 / \mathrm{x}}$ & 67 & 42 & 25 & $0.003^{* *}$ & 57 & 10 & $<0.001^{* * *}$ \\
\hline & $\mathrm{N}_{+}$ & 47 & 16 & 31 & & 16 & 31 & \\
\hline \multirow[t]{2}{*}{ SCC-Ag (ng/mL) } & $<1.5$ & 50 & 25 & 25 & 0.911 & 39 & 11 & $0.002 * *$ \\
\hline & $\geq 1.5$ & 43 & 21 & 22 & & 20 & 23 & \\
\hline \multirow[t]{2}{*}{$\mathrm{CRP}(\mathrm{mg} / \mathrm{mL})$} & $<5.0$ & 57 & 31 & 26 & 0.283 & 40 & 17 & $0.041^{*}$ \\
\hline & $\geq 5.0$ & 22 & 9 & 13 & & 10 & 12 & \\
\hline \multirow[t]{2}{*}{ sLAMC2 (ng/mL) } & $<108$ & 58 & 48 & 10 & $<0.00 I^{* * *}$ & - & - & - \\
\hline & $\geq 108$ & 56 & 25 & 31 & & - & - & \\
\hline
\end{tabular}

Notes: $* P<0.05 ; * * P<0.01 ; * * * P<0.001$.

Abbreviations: CRP, C-reactive protein; LAMC2, laminin gamma 2; PSCC, penile squamous cell carcinoma; SCC-Ag, squamous cell carcinoma antigen; sLAMC2, serum LAMC2 level; tLAMC2, tissue LAMC2 level. 

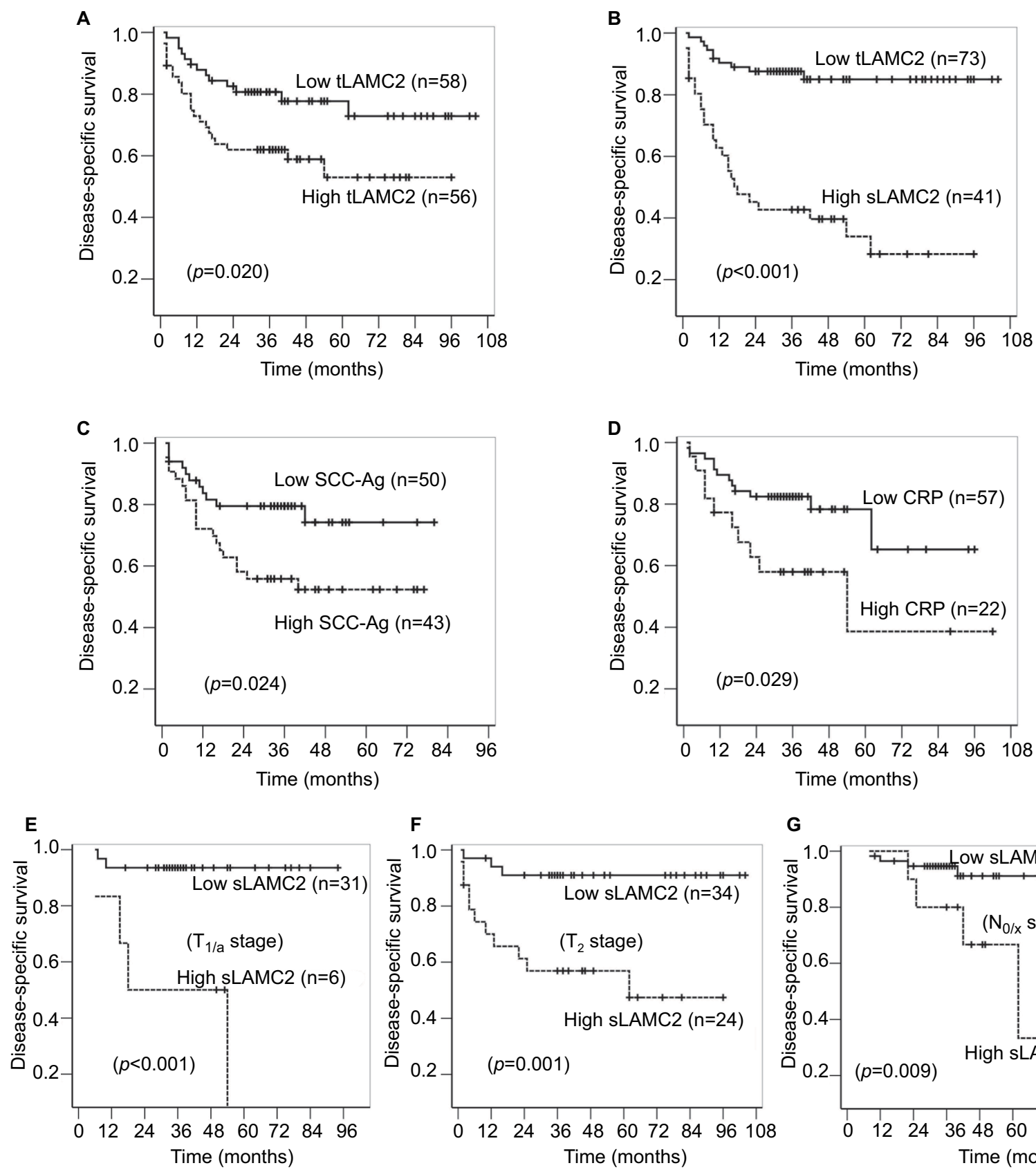

$\mathbf{F}$

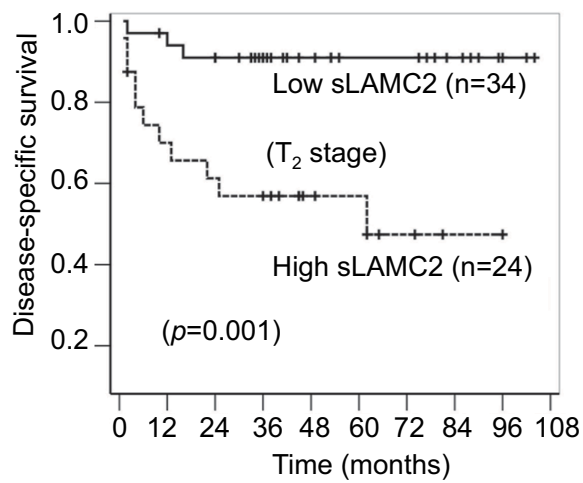

G

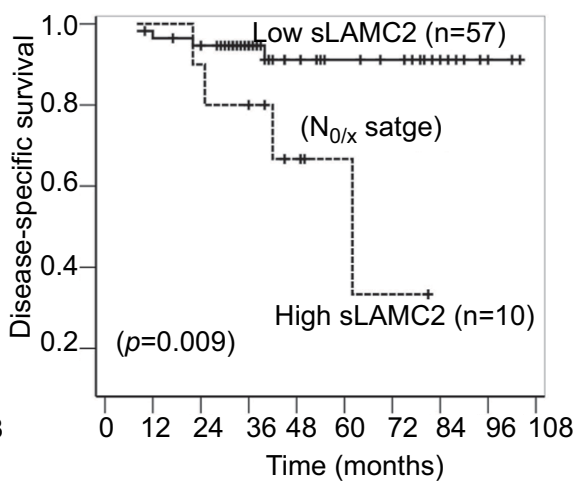

Figure 3 The analyses for disease-specific survival in PSCC patients.

Notes: (A-B): Kaplan-Meier survival analyses were performed on the patient cohort based on the levels of tLAMC2 (A), sLAMC2 (B), SCC-Ag (C), and CRP (D). (E-G): Stratified Kaplan-Meier survival analyses based on sLAMC2 level in patients at $T_{1 / a}(E), T_{2}(F)$, and $N_{0 / x}(\mathbf{G})$ stage.

Abbreviations: CRP, C-reactive protein; LAMC2, laminin gamma 2; PSCC, penile squamous cell carcinoma; SCC-Ag, squamous cell carcinoma antigen; sLAMC2, serum LAMC2 level; tLAMC2, tissue LAMC2 level.

In previous reports, elevated expression of $L A M C 2$ was detected in numerous malignancies, ${ }^{17-19}$ and cytoplasmic accumulation of LAMC2 and/or an increase in serum LAMC2 was correlated with tumor metastasis and poor prognosis in patients. ${ }^{12,18,19}$ Here, we found that $L A M C 2$ expression was upregulated, LAMC2 demonstrated an especially high cytoplasmic accumulation in PSCC tissues, and that LAMC2 overexpression was associated with LN metastasis in PSCC. Furthermore, sLAMC2 level was associated with $\mathrm{N}$ stage and pathologic grade in the analyzed patients. The relationship between LAMC2 level and N stage in PSCC patients was also supported by the results of our in vitro and in vivo experiments, which indicated that LAMC2 promoted PSCC cell metastasis. The effects of 
Table 2 Multivariate Cox proportional hazards regression analysis for DSS

\begin{tabular}{|c|c|c|c|c|c|c|}
\hline \multirow[t]{2}{*}{ Variables } & \multicolumn{3}{|c|}{ Model I } & \multicolumn{3}{|c|}{ Model 2} \\
\hline & HR & $95 \% \mathrm{Cl}$ & $P$-value & HR & $95 \% \mathrm{Cl}$ & $P$-value \\
\hline $\mathrm{T}$ stage $\left(\mathrm{T}_{2-4}\right.$ vs $\left.\mathrm{T}_{1 / \mathrm{a}}\right)$ & 2.148 & $0.887-5.200$ & 0.090 & 1.882 & $0.773-4.584$ & 0.164 \\
\hline$G$ grade $\left(G_{2-3}\right.$ vs $\left.G_{1}\right)$ & 2.405 & I.158-4.995 & $0.019 *$ & 2.252 & $1.075-4.719$ & $0.03 I^{*}$ \\
\hline $\mathrm{N}$ stage $\left(\mathrm{N}_{+}\right.$vs $\left.\mathrm{N}_{0 / \mathrm{X}}\right)$ & 5.157 & $2.263-11.747$ & $<0.001 * * *$ & 3.375 & I.352-8.425 & $0.009 * *$ \\
\hline tLAMC2 ( $\geq 30 \%$ vs $<30 \%)$ & 1.292 & $0.640-2.607$ & 0.474 & - & - & - \\
\hline sLAMC2 (ng/mL) ( $\geq 108$ vs $<108)$ & - & - & - & 2.780 & $1.219-6.34 \mid$ & $0.015^{*}$ \\
\hline
\end{tabular}

Notes: $* P<0.05 ; * * P<0.01 ; * * * P<0.001$.

Abbreviations: DSS, disease-special survival; LAMC2, laminin gamma 2; sLAMC2, serum LAMC2 level; tLAMC2, tissue LAMC2 level.

Table 3 Stratified analysis for DSS

\begin{tabular}{|c|c|c|c|c|}
\hline Subgroups & No. of patients & $\begin{array}{l}\text { Mean DSS (months) } \\
(\text { sLAMC2 } \\
\left.\text { high } \text { vs sLAMC2 }^{\text {low }}\right)\end{array}$ & $P$-value & HR (95\% Cl) \\
\hline All patients & 114 & 40.4 vs 90.7 & $<0.00 I^{* * * *}$ & $6.37(3.06-13.24)$ \\
\hline \multicolumn{5}{|l|}{ T stage } \\
\hline$T_{1 / a}$ & 37 & 33.5 vs 88.5 & $0.003^{* *}$ & $|2.9|(2.35-70.9 \mid)$ \\
\hline $\mathrm{T}_{2}$ & 58 & 55.3 vs 95.5 & $0.004^{* *}$ & $6.46(1.80-23.21)$ \\
\hline $\mathrm{T}_{3 / 4}^{2}$ & 19 & 11.8 vs 25.8 & 0.101 & $2.53(0.84-7.65)$ \\
\hline \multicolumn{5}{|c|}{ Pathologic grade } \\
\hline $\mathrm{G}_{1}$ & 70 & 59.7 vs 96.8 & $0.002^{* *}$ & $6.49(1.95-21.60)$ \\
\hline $\mathrm{G}_{2}$ & 31 & 29.5 vs 82.3 & $0.012 *$ & $6.79(1.53-30.18)$ \\
\hline $\mathrm{G}_{3 / 4}$ & 13 & 4.40 vs 46.91 & $0.014 *$ & $8.38(1.53-45.76)$ \\
\hline \multicolumn{5}{|l|}{$\mathrm{N}$ stage } \\
\hline$N_{0 / x}$ & 67 & 58.0 vs 97.0 & $0.020 *$ & $5.26(|.3|-2 \mid .22)$ \\
\hline $\mathrm{N}_{1 / 2}$ & 20 & 52.3 vs 84.0 & 0.148 & $4.78(0.57-39.83)$ \\
\hline $\mathrm{N}_{3}$ & 27 & I 4.6 vs 25.4 & 0.249 & $1.81(0.66-4.96)$ \\
\hline
\end{tabular}

Notes: $* P<0.05 ; * * P<0.01 ; * * * P<0.001$.

Abbreviations: DSS, disease-special survival; LAMC2, laminin gamma 2; sLAMC2, serum LAMC2 level.

LAMC2 on PSCC cells may partially result from LAMC2directed EMT in PSCC cells; this hypothesis is supported by previous reports showing that EMT endows cells with migratory and invasive properties. ${ }^{23}$

Elevated levels of both tLAMC2 and sLAMC2 were significantly associated with shorter DSS in PSCC patients; however, N stage, pathologic grade, and SLAMC2, but not tLAMC2, SCC-Ag, or CRP, were independent prognostic factors according to the multivariate regression analysis in our study. These results may be in accordance with the lower HRs for tLAMC2, SCC-Ag, or CRP compared to those for $\mathrm{N}$ stage, pathologic grade, and sLAMC2 (Table S4). Thus, we speculate that $\mathrm{SLAMC} 2$ is a more important predictive factor for survival in PSCC patients than SCC-Ag and CRP, which have been previously reported to be potential predictive factors in PSCC patients. In particular, the stratification analysis revealed that sLAMC2 level showed more predictive value for DSS in patients with early-stage penile cancer (such as patients at the $\mathrm{N}_{0 / \mathrm{X}}$ stage). However, due to the lower number of patients at the $T_{3 / 4}$ stage in our analysis, we cannot conclude that sLAMC2 level showed more predictive value in early T-stage penile cancer, and this finding needs to be further investigated in a large cohort.

According to the NCCN Guidelines for Penile Cancer (Version 2.2018), up to $25 \%$ of penile cancer patients with nonpalpable LNs harbor micrometastases and several predictive factors and risk stratification systems have been evaluated for the prediction of the presence of occult node metastasis. ${ }^{36}$ However, these factors and systems are based on pathologic diagnosis, which may introduce errors because of the heterogeneity of tumors and the limitations of sampling. Our findings in this study suggested that serum LAMC2 level might be useful for risk stratification in these patients and might add prognostic information to the conventional clinicopathologic risk factors.

However, some barriers must be overcome in the development of sLAMC2 as a serologic index from the bench to the clinic. First, independent, multicenter, large-scale retrospective studies will be necessary. Next, prospective controlled clinical trials are also needed before this index can be brought into clinical use, especially for $\mathrm{LN}$ metastasis prediction and treatment monitoring. There are some limitations in our 
study: 1) only a small sample of PSCC patients from a single center was included, so selection bias is inevitable; 2) the number of patients at an advanced $\mathrm{T}$ stage $\left(\mathrm{T}_{3 / 4}\right)$ or with $\mathrm{LN}$ metastasis $\left(\mathrm{N}_{+}\right)$was lower than the number of patients at the $\mathrm{T}_{1 / 2}$ or $\mathrm{N}_{0}$ stage, so we could not confirm that sLAMC2 level does not have predictive value for DSS in PSCC patients at a high $\mathrm{T}$ stage $\left(\mathrm{T}_{3 / 4}\right)$ or with $\mathrm{LN}$ metastasis $\left(\mathrm{N}_{+}\right)$; and 3$)$ the diversity of adjuvant therapy may also affect the analysis. Given the deficiencies mentioned above, all of the analyses in this study were regarded as hypothesis generating rather than hypothesis testing.

\section{Conclusion}

Our study revealed that LAMC2 plays a critical role in PSCC progression and metastasis; elevated SLAMC2 levels were strongly correlated with advanced clinicopathologic parameters, especially LN metastasis, in PSCC patients; SLAMC2 level might serve as a potential prognostic biomarker in PSCC patients, especially in those with early-stage penile cancer (especially patients at the $\mathrm{N}_{0 / \mathrm{X}}$ stages); the predictive value of sLAMC2 level is superior to that of CRP and SCC-Ag previously reported in PSCC patients. Certainly, studies based on large samples from multiple centers are needed to assess the possibility of clinical application of LAMC2.

\section{Acknowledgments}

The authors thank Dr Xinke Zhang and Dr Shumei Yan (Department of Pathology, SYSUCC) for IHC scoring of LAMC2 and E-cadherin. We also thank Dr Jibin Li (Clinical Research Unit, SYSUCC) for guidance in the statistical analyses. The authenticity of this article was validated by uploading the key raw data into the Research Data Deposit public platform (www.researchdata.org.cn) with the approval number RDDB2018000359. This study was supported by the National Natural Science Foundation of China (81772755, 81472256), the Guangdong Provincial Science and Technology Project (2015A030302018, 2016A020215081), and the Guangzhou Municipal Science and Technology Project (201704030037).

\section{Disclosure}

The authors report no conflicts of interest in this work.

\section{References}

1. Hakenberg OW, Compérat EM, Minhas S, Necchi A, Protzel C, Watkin N. EAU guidelines on penile cancer: 2014 update. Eur Urol. 2015;67(1):142-150.

2. Spiess PE, Dhillon J, Baumgarten AS, Johnstone PA, Giuliano AR. Pathophysiological basis of human papillomavirus in penile cancer: key to prevention and delivery of more effective therapies. CA Cancer $J$ Clin. 2016;66(6):481-495.
3. Li ZS, Yao K, Chen $\mathrm{P}$, et al. Modification of $\mathrm{N}$ staging systems for penile cancer: a more precise prediction of prognosis. Br J Cancer. 2015;112(11):1766-1771.

4. Ornellas AA, Kinchin EW, Nóbrega BL, Wisnescky A, Koifman N, Quirino R. Surgical treatment of invasive squamous cell carcinoma of the penis: Brazilian National Cancer Institute long-term experience. $J$ Surg Oncol. 2008;97(6):487-495.

5. Yao K, Tu H, Li YH, Qin ZK, Liu ZW, Zhou FJ, Han H. Modified technique of radical inguinal lymphadenectomy for penile carcinoma: morbidity and outcome. J Urol. 2010;184(2):546-552.

6. Sharma P, Djajadiningrat R, Zargar-Shoshtari K, et al. Adjuvant chemotherapy is associated with improved overall survival in pelvic nodepositive penile cancer after lymph node dissection: a multi-institutional study. Urol Oncol. 2015;33(11):e417-496.

7. Li ZS, Deng CZ, Yao K, et al. Bilateral pelvic lymph node dissection for Chinese patients with penile cancer: a multicenter collaboration study. J Cancer Res Clin Oncol. 2017;143(2):329-335.

8. Diorio GJ, Leone AR, Spiess PE. Management of penile cancer. Urology. 2016;96:15-21.

9. Zargar-Shoshtari K, Sharma P, Djajadiningrat R, et al. Extent of pelvic lymph node dissection in penile cancer may impact survival. World $J$ Urol. 2016;34(3):353-359.

10. Zamcheck N, Pusztaszeri G, Cea PG. CEA, AFP and other potential tumor markers. CA Cancer J Clin. 1975;25(4):204-214.

11. Brawer MK, Lange $P H$. Prostate-specific antigen and premalignant change: implications for early detection. CA Cancer J Clin. 1989;39(6):361-375.

12. Chan A, Prassas I, Dimitromanolakis A, et al. Validation of biomarkers that complement CA19.9 in detecting early pancreatic cancer. Clin Cancer Res. 2014;20(22):5787-5795.

13. Li ZS, Yao K, Li YH, et al. Clinical significance of preoperative C-reactive protein and squamous cell carcinoma antigen levels in patients with penile squamous cell carcinoma. BJU Int. 2016;118(2):272-278.

14. Zhu Y, Ye DW, Yao XD, et al. The value of squamous cell carcinoma antigen in the prognostic evaluation, treatment monitoring and followup of patients with penile cancer. J Urol. 2008;180(5):2019-2023.

15. Kommu S, Hadway P, Watkin N. Squamous cell carcinoma antigen as a biomarker for penile cancer. BJU Int. 2005;95(4):478-479.

16. Fukushima Y, Ohnishi T, Arita N, Hayakawa T, Sekiguchi K. Integrin alpha3beta1-mediated interaction with laminin-5 stimulates adhesion, migration and invasion of malignant glioma cells. Int J Cancer. 1998;76(1):63-72.

17. Yamamoto H, Itoh F, Iku S, Hosokawa M, Imai K. Expression of the gamma(2) chain of laminin-5 at the invasive front is associated with recurrence and poor prognosis in human esophageal squamous cell carcinoma. Clin Cancer Res. 2001;7(4):896-900.

18. Takahashi S, Hasebe T, Oda T, et al. Cytoplasmic expression of laminin gamma2 chain correlates with postoperative hepatic metastasis and poor prognosis in patients with pancreatic ductal adenocarcinoma. Cancer. 2002;94(6):1894-1901.

19. Korbakis D, Dimitromanolakis A, Prassas I, et al. Serum LAMC2 enhances the prognostic value of a multi-parametric panel in non-small cell lung cancer. Br J Cancer. 2015;113(3):484-491.

20. Fu X, Hu J, Han HY, et al. High expression of XPA confers poor prognosis for nasopharyngeal carcinoma patients treated with platinum-based chemoradiotherapy. Oncotarget. 2015;6(29):28478-28490.

21. Geng R, Tan X, Zuo Z, et al. Synthetic lethal short hairpin RNA screening reveals that ring finger protein 183 confers resistance to trametinib in colorectal cancer cells. Chin J Cancer. 2017;36(1):63.

22. Smith SC, Nicholson B, Nitz M, et al. Profiling bladder cancer organ site-specific metastasis identifies LAMC2 as a novel biomarker of hematogenous dissemination. Am J Pathol. 2009;174(2):371-379.

23. Masferrer E, Ferrándiz-Pulido C, Masferrer-Niubò M, et al. Epithelialto-mesenchymal transition in penile squamous cell carcinoma. J Urol. 2015;193(2):699-705

24. Liu RY, Dong Z, Liu J, et al. Overexpression of asparagine synthetase and matrix metalloproteinase 19 confers cisplatin sensitivity in nasopharyngeal carcinoma cells. Mol Cancer Ther. 2013;12(10): 2157-2166. 
25. Liu RY, Dong Z, Liu J, et al. Role of eIF3a in regulating cisplatin sensitivity and in translational control of nucleotide excision repair of nasopharyngeal carcinoma. Oncogene. 2011;30(48):4814-4823.

26. Liu RY, Wu LZ, Huang BJ, et al. Adenoviral expression of a truncated S1 subunit of SARS-CoV spike protein results in specific humoral immune responses against SARS-CoV in rats. Virus Res. 2005;112(1-2):24-31.

27. Li ZH, Tang QB, Wang J, et al. Hepatitis $\mathrm{C}$ virus core protein induces malignant transformation of biliary epithelial cells by activating nuclear factor-kappaB pathway. J Gastroenterol Hepatol. 2010;25(7):1315-1320.

28. Zhou QH, Deng CZ, Li ZS, et al. Molecular characterization and integrative genomic analysis of a panel of newly established penile cancer cell lines. Cell Death Dis. 2018;9(6):684.

29. Chen J, Yao K, Li Z, et al. Establishment and characterization of a penile cancer cell line, penl1, with a deleterious TP53 mutation as a paradigm of HPV-negative penile carcinogenesis. Oncotarget. 2016;7(32): 51687-51698.

30. Deng C, Li Z, Guo S, et al. Tumor PD-L1 expression is correlated with increased TILs and poor prognosis in penile squamous cell carcinoma. Oncoimmunology. 2017;6(2):e1269047.
31. Li Y, Ye Z, Chen S, et al. ARHGEF19 interacts with BRAF to activate MAPK signaling during the tumorigenesis of non-small cell lung cancer. Int J Cancer. 2018;142(7):1379-1391.

32. Kuang CM, Fu X, Hua YJ, et al. BST2 confers cisplatin resistance via NF- $\kappa \mathrm{B}$ signaling in nasopharyngeal cancer. Cell Death Dis. 2017;8(6):e2874.

33. Liu RY, Zhou L, Zhang YL, et al. An oncolytic adenovirus enhances antiangiogenic and antitumoral effects of a replication-deficient adenovirus encoding endostatin by rescuing its selective replication in nasopharyngeal carcinoma cells. Biochem Biophys Res Commun. 2013;442(3-4): 171-176.

34. Du WY, Lu ZH, Ye W, et al. The loss-of-function mutations and downregulated expression of ASB3 gene promote the growth and metastasis of colorectal cancer cells. Chin J Cancer. 2017;36(1):11.

35. Zhou Y, Wu J, Fu X, et al. OTUB1 promotes metastasis and serves as a marker of poor prognosis in colorectal cancer. Mol Cancer. 2014;13: 258.

36. NCCN. Clinical Practice Guidelines in Oncology (NCCN Guidelines): Penile Cancer. Version 2; 2018; MS-8. 


\section{Supplementary materials}

Table SI Clinicopathologic characteristics ${ }^{\mathrm{a}}$

\begin{tabular}{|c|c|c|c|}
\hline Variables & & Number of patients & $\%$ \\
\hline \multirow[t]{3}{*}{ Age (years) } & Median (range) & $54(24-86)$ & \\
\hline & $<54$ & 57 & 50.0 \\
\hline & $\geq 54$ & 57 & 50.0 \\
\hline \multirow[t]{2}{*}{ Phimosis } & No & 33 & 28.9 \\
\hline & Yes & 81 & 71.1 \\
\hline \multirow[t]{4}{*}{ T stage } & $T_{1 / a}$ & 37 & 33.5 \\
\hline & $\mathrm{T}_{2}$ & 58 & 50.9 \\
\hline & $\mathrm{T}_{3}$ & 12 & 10.5 \\
\hline & $\mathrm{T}_{4}$ & 7 & 6.1 \\
\hline \multirow[t]{3}{*}{ Pathology grade } & $\mathrm{G}_{1}$ & 70 & 61.4 \\
\hline & $\mathrm{G}_{2}$ & 31 & 27.2 \\
\hline & $\mathrm{G}_{3}$ & 13 & 11.4 \\
\hline \multirow[t]{4}{*}{$N$ stage } & $N_{0 / x}$ & 67 & 58.8 \\
\hline & $N_{1}$ & 11 & 9.6 \\
\hline & $\mathrm{N}_{2}$ & 9 & 7.9 \\
\hline & $\mathrm{N}_{3}$ & 27 & 23.7 \\
\hline \multirow[t]{2}{*}{ SCC-Ag (ng/mL) } & $<1.5$ & 50 & 53.8 \\
\hline & $\geq 1.5$ & 43 & 46.2 \\
\hline \multirow[t]{2}{*}{$\mathrm{CRP}(\mathrm{mg} / \mathrm{mL})$} & $<5.0$ & 57 & 72.2 \\
\hline & $\geq 5.0$ & 22 & 27.8 \\
\hline \multirow[t]{2}{*}{ sLAMC2 (ng/mL) } & $<108$ & 73 & 64.0 \\
\hline & $\geq 108$ & 41 & 36.0 \\
\hline \multirow[t]{2}{*}{ tLAMC2 } & $\leq 30 \%$ & 58 & 50.9 \\
\hline & $>30 \%$ & 56 & 49.1 \\
\hline
\end{tabular}

Table S2 Sequences of the primers used in this paper

\begin{tabular}{llll}
\hline Gene & Direction & Primer sequences & Usage \\
\hline LAMC2 & Forward & $5^{\prime}$-GGAATTCACCATGCCTGCGCTCTGGCTGGGC-3' & cDNA cloning \\
& Reverse & $5^{\prime}$-CGCGGATCCCTGTTGCTCAAGAGCCTGGGT-3' & \\
LAMC2 & Forward & $5^{\prime}$-GATGGCATTCACTGCGAGAAG-3' & Quantitative PCR \\
& Reverse & $5^{\prime}$-TCGAGCACTAAGAGAACCTTTGG-3' & \\
GAPDH & Forward & $5^{\prime}$-GACTCATGACCACAGTCCATGC-3' & Quantitative PCR \\
& Reverse & $5^{\prime}$-AGAGGCAGGGATGATGTTCTG-3' & \\
\hline
\end{tabular}

Abbreviation: LAMC2, laminin gamma 2.

Table S3 Target sequences of siRNAs and shRNAs used in this paper

\begin{tabular}{lll}
\hline Name & Target genes & Target sequence \\
\hline shl & LAMC2 & 5'-GCAGAATACAGTGTCCATA-3' \\
sh2 & LAMC2 & 5'-GCAGGTGTTTGAAGTGTAT-3' \\
shNC & Negative control & 5'-TTCTCCGAACGTGTCACGT-3' \\
\hline
\end{tabular}

Abbreviation: LAMC2, laminin gamma 2. 
Table S4 Univariate Cox proportional hazards regression analyses for DSS

\begin{tabular}{|c|c|c|c|c|}
\hline Variables & No. of patients & HR & $95 \% \mathrm{Cl}$ & $P$-value \\
\hline Age (years) ( $\geq 54$ vs <54) & 114 & 1.018 & $0.530-1.958$ & 0.957 \\
\hline Phimosis (yes vs no) & 114 & 0.914 & $0.646-1.293$ & 0.613 \\
\hline T stage $\left(T_{2-4}\right.$ vs $\left.T_{1 / a}\right)$ & 114 & 2.772 & $1.153-6.663$ & $0.023^{*}$ \\
\hline Pathology grade $\left(G_{2-3}\right.$ vs $\left.G_{1}\right)$ & 114 & 4.055 & $2.023-8.125$ & $<0.00 I^{* *}$ \\
\hline$N$ stage $\left(N_{+}\right.$vs $\left.N_{0 / X}\right)$ & 114 & 7.628 & $3.46|-| 6.8 \mid 4$ & $<0.00 I^{* *}$ \\
\hline SCC-Ag (ng/mL) $(\geq 1.5$ vs $<1.5)$ & 93 & 2.269 & $1.087-4.737$ & $0.029 *$ \\
\hline $\operatorname{CRP}(\mathrm{mg} / \mathrm{mL})(\geq 5.0$ vs $<5.0)$ & 79 & 2.465 & $1.064-5.712$ & $0.035^{*}$ \\
\hline sLAMC2 (ng/mL) ( $\geq 108$ vs < 108$)$ & 114 & 6.370 & $3.064-13.242$ & $<0.00 I^{* *}$ \\
\hline tLAMC2 (>30\% vs $\leq 30 \%)$ & 114 & 2.185 & $1.105-4.319$ & $0.025^{*}$ \\
\hline
\end{tabular}

Notes: $* P<0.05, * * P<0.01$.

Abbreviations: CRP, C-reactive protein; DSS, disease-special survival; LAMC2, laminin gamma 2; SCC-Ag, squamous cell carcinoma antigen; sLAMC2, serum LAMC2 level; tLAMC2, tissue LAMC2 level.

Table S5 Multivariate Cox proportional hazards regression analyses for DSS

\begin{tabular}{|c|c|c|c|c|c|c|}
\hline \multirow[t]{2}{*}{ Variables } & \multicolumn{3}{|c|}{ Model 3} & \multicolumn{3}{|c|}{ Model 4} \\
\hline & $\overline{\mathbf{H R}}$ & $95 \% \mathrm{Cl}$ & $P$-value & HR & $95 \% \mathrm{Cl}$ & $P$-value \\
\hline$T$ stage $\left(T_{2-4}\right.$ vs $\left.T_{1 / a}\right)$ & 3.142 & $1.080-9.145$ & $0.036 *$ & 3.490 & $0.995-12.243$ & 0.051 \\
\hline$G$ grade $\left(G_{2-3}\right.$ vs $\left.G_{1}\right)$ & 3.114 & $1.410-6.878$ & $0.005^{* *}$ & 3.200 & $1.180-8.677$ & $0.022 *$ \\
\hline$N$ stage $\left(N_{+}\right.$vs $\left.N_{0 / x}\right)$ & 3.881 & $1.562-9.645$ & $0.004 * *$ & 5.190 & $1.817-14.825$ & $0.002 * *$ \\
\hline SCC-Ag (ng/mL) $(\geq 1.5$ vs $<1.5)$ & 1.230 & $0.574-2.634$ & 0.594 & - & - & - \\
\hline $\mathrm{CRP}(\mathrm{mg} / \mathrm{mL})(\geq 5.0$ vs $<5.0)$ & - & - & - & 1.820 & $0.763-4.343$ & 0.177 \\
\hline
\end{tabular}

Notes: $* P<0.05, * * P<0.01$.

Abbreviations: CRP, C-reactive protein; DSS, disease-special survival; SCC-Ag, squamous cell carcinoma antigen. 

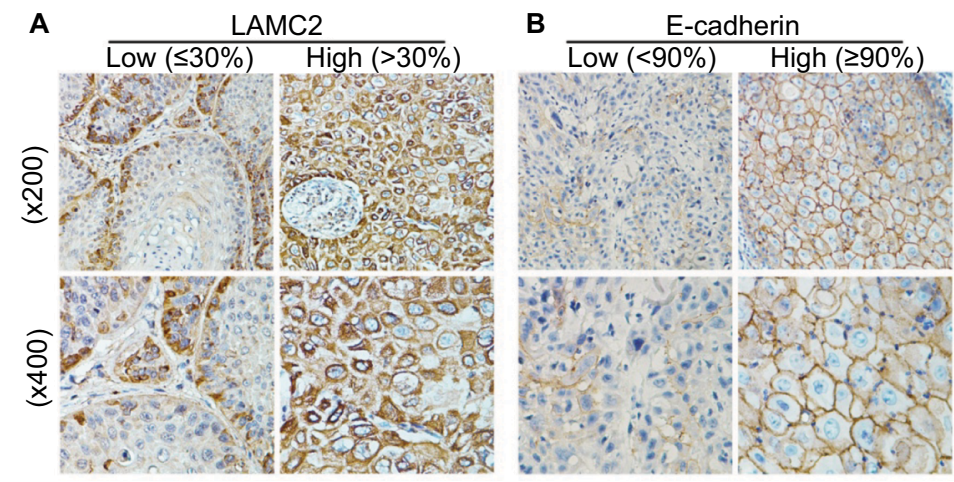

Figure SI Immunohistochemistry assay for the expression of $L A M C 2$ and E-cadherin in PSCC tissues.

Note: Representative pictures.

Abbreviations: LAMC2, laminin gamma 2; PSCC, penile squamous cell carcinoma.

A

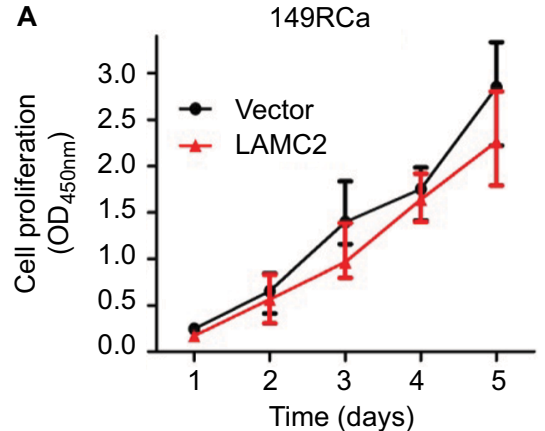

B

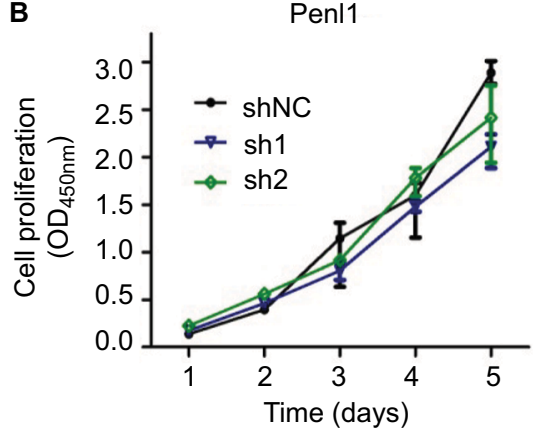

C

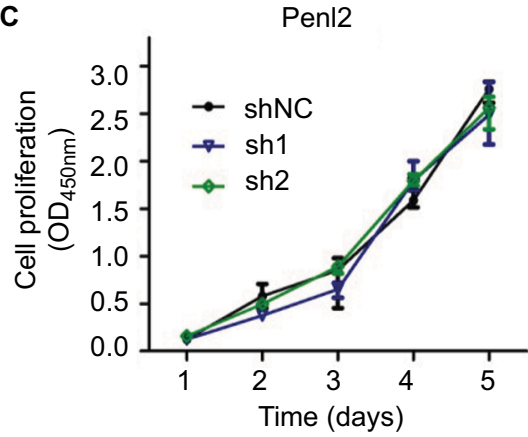

Figure S2 Effect of LAMC2 on penile squamous cell carcinoma cell proliferation.

Note: Cell proliferation was analyzed using CCK-8 in I49RCa (A), Penll (B), and Penl2 (C).

Abbreviations: LAMC2, laminin gamma 2; shI, sh2: LAMC2 shRNAs; shNC, negative control shRNA; OD, optical density.

Cancer Management and Research

\section{Publish your work in this journal}

Cancer Management and Research is an international, peer-reviewed open access journal focusing on cancer research and the optimal use of preventative and integrated treatment interventions to achieve improved outcomes, enhanced survival and quality of life for the cancer patient. The manuscript management system is completely online and includes

\section{Dovepress}

a very quick and fair peer-review system, which is all easy to use. Visit http://www.dovepress.com/testimonials.php to read real quotes from published authors. 